\title{
Erratum to: Improved superaugmented eccentric connectivity indices for QSAR/QSPR part I: development and evaluation
}

\author{
Rohit Dutt · A. K. Madan
}

Published online: 8 May 2010

(C) Springer Science+Business Media, LLC 2010

\section{Erratum to: Med Chem Res \\ DOI 10.1007/s00044-009-9200-1}

Due to typographical error, this paper published online with incorrect data in Table 2. The corrected of version Table 2 is as follows.

The online version of the original article can be found under doi:10.1007/s00044-009-9200-1.

R. Dutt

Guru Gobind Singh College of Pharmacy, Yamunanagar 135001, India

A. K. Madan ( $\bowtie)$

Faculty of Pharmaceutical Sciences, M. D. University, Rohtak 124001, India

e-mail: madan_ak@yahoo.com 
Table 2 Comparison of discriminating power and degeneracy of proposed TIs using various structures with three, four and five vertices

\begin{tabular}{llllllll}
\hline & $\xi^{\mathrm{c}}$ & $A^{\mathrm{c}}$ & $S A \xi_{3}^{\mathrm{c}}$ & $S A \xi_{4}^{\mathrm{c}}$ & $S A \xi_{5}^{\mathrm{c}}$ & $S A \xi_{6}^{\mathrm{c}}$ & $S A \xi_{7}^{\mathrm{c}}$ \\
\hline For three vertices & & & & & & & \\
Minimum value & 6 & 3 & 1.25 & 5 & 3 & 2 & 1.5 \\
Maximum value & 6 & 12 & 12 & 48 & 48 & 48 & 48 \\
Ratio & $1: 1$ & $1: 4$ & $1: 9.6$ & $1: 9.6$ & $1: 16$ & $1: 24$ & $1: 32$ \\
Degeneracy & $1 / 2$ & $0 / 2$ & $0 / 2$ & $0 / 2$ & $0 / 2$ & $0 / 2$ & $0 / 2$ \\
For four vertices & & & & & & & 0.60 \\
Minimum value & 9 & 3.33 & 0.3 & 6.67 & 2.89 & 1.30 & 2916 \\
Maximum value & 16 & 108 & 108 & 2916 & 2916 & 2916 & $1: 4870$ \\
Ratio & $1: 1.78$ & $1: 32.4$ & $1: 360.7$ & $1: 437.4$ & $1: 1009.38$ & $1: 2249$ & $0 / 6$ \\
Degeneracy & $1 / 6$ & $0 / 6$ & $0 / 6$ & $0 / 6$ & $0 / 6$ & $0 / 6$ & \\
For five vertices & & & & & & & 1.08 \\
Minimum value & 12 & 4.33 & 0.32 & 12.67 & 5.39 & 2.42 & 327680 \\
Maximum value & 28 & 1280 & 1280 & 327680 & 327680 & 327680 & $1: 303407$ \\
Ratio & $1: 2.34$ & $1: 295.4$ & $1: 4063$ & $1: 25869$ & $1: 60807$ & $1: 135332$ & $1 / 21$ \\
Degeneracy & $11 / 21$ & $0 / 21$ & $0 / 21$ & $0 / 21$ & $0 / 21$ & $0 / 21$ & \\
\hline
\end{tabular}

Degeneracy $=$ Number of compounds having same values/total number of compounds with same number of vertices 